\title{
An Evidence Based Approach to the First Unprovoked Seizure
}

\author{
Samuel Wiebe
}

\begin{abstract}
The objective is to illustrate the creation and structure of a particular type of Evidence Based Care (EBC) summary that has direct clinical relevance, the Critically Appraised Topic (CAT). The process consists of a step-by-step application of the EBC principles to a common neurological problem, ie., a patient presenting with a first, unprovoked generalized seizure. This includes asking a focused clinical question about prognosis for recurrence and the role of antiepileptic drugs; searching the literature to answer the question; selecting the relevant evidence (a meta-analysis about prognosis and a randomized controlled trial about therapy); appraising the literature for its validity and usefulness; and applying the results to the clinical scenario. The result is a one-page, user friendly CAT whose title states a declarative answer to the clinical question. It also contains a description of the literature search and of the evidence, the clinical bottom lines derived from the evidence, and general comments.
\end{abstract}

RÉSUMÉ: Une approche basée sur des preuves à la prise en charge d'une première crise convulsive non provoquée. L'objectif de cet article est d'illustrer la création et la structure d'un type particulier de sommaire de soins fondés sur des preuves (evidence-based care - EBC) ayant une pertinence clinique directe, le critically appraised topic (CAT). Le processus consiste à appliquer étape par étape les principes d'EBC à un problème neurologique fréquent, i.e. celui d'un patient qui consulte pour une première crise convulsive généralisée non provoquée. Ce processus inclut: des questions cliniques ciblées sur le pronostic de récidive et le rôle des antiépileptiques; une recherche de la littérature pour répondre aux questions; la sélection des données pertinentes (une méta-analyse sur le pronostic et un essai clinique contrôlé et randomisé sur le traitement); l'évaluation de la littérature quant à sa validité et à son utilité; et l'application des résultats au scénario clinique. Le résultat est un CAT de deux pages, facile à utiliser, dont le titre énonce une réponse à la question clinique. Il contient également une description de la recherche de la littérature et des données, des conclusions cliniques tirées des données et des commentaires généraux.

Can. J. Neurol. Sci. 2002; 29: 120-124

There are few sources of valid, Evidence Based Care (EBC) summaries on which clinicians in the neurosciences can draw to assist them in moving towards EBC (see article in this issue). The critically appraised topic (CAT) is a concise, user-friendly, EBC summary that can help clinicians'needs for relevant, easily accessible EBC information, and assist in clinical decision making. A collection of updateable, peer-reviewed CATs covering a wide breadth of neurosciences topics, can help clinicians implement EBC without having to engage in the entire EBC process. Focusing on a specific clinical question, ie, management of patients presenting with a first unprovoked seizure, we illustrate the principles of EBC, how a CAT is generated, and provide a one-page CAT for this question.

\section{THE CLINICAL QUESTIONS}

A previously-healthy, 25-year-old female, university student presents with a witnessed, first-ever, generalized tonic clonic seizure lasting two to three minutes, followed by a deep sleep for
15 minutes and transient generalized headache. A detailed history reveals no warning for her seizure, nor any ictal or post ictal lateralising features, and there are no precipitating factors or pathological antecedents. Her neurological examination is normal. She volunteers that her brother has well-controlled grand mal seizures. Her CBC and biochemistry are normal and you decide to obtain an EEG and a head CTand to review her and her test results. She wants to know what her risk is of having another seizure and whether she should take anticonvulsants.

This clinical encounter generates several clinical questions. For example, what is the differential diagnosis of this event? What is the diagnostic and prognostic usefulness of EEG, and

From the Department of Clinical Neurological Sciences, University of Western Ontario, and the London Health Sciences Centre London, Ontario, Canada Receivedseptember 13, 2001. AcCePtedin FINAL FORM DeCEMBER 13, 2001 Reprint requests to: Samuel Wiebe, London Health Sciences Centre, University Campus, 339 Windermere Rd., London, Ontario, Canada N6A5A5 
imaging? What is the yield of routine blood work? What is the prognosis for recurrence? Should you recommend antiepileptic drug (AED) treatment? What about driving? Evidence based care can help us conscientiously and judiciously apply the best external evidence to the management of this patient with a first generalized tonic clonic seizure.

\section{THE EBC PROCESS}

The EBC process entails stating the clinical problem at hand in the form of a defined, answerable question; efficiently searching the literature for the best evidence; critically appraising the evidence for its validity and usefulness; and applying the evidence in the context of the patients' circumstances and values. We will address each step in turn.

\section{Formulating a focused clinical question}

In order to find and apply the evidence to the care of individual patients, information needs must be turned into focused questions. For this analysis we will focus on prognosis, and on the role of anticonvulsants after a first, unprovoked, generalized seizure. Focused, answerable questions typically have three components:

1) The patient (a healthy woman with a single, unprovoked generalized tonic clonic seizure);

2) The intervention (the passage of time if we are interested in prognosis, or anticonvulsants in the case of therapy), and

3) The outcome (seizure recurrence).

\section{Finding the evidence}

Books and traditional reviews give some guidance for the management of first unprovoked seizures and comment on the risk of recurrence. However, these sources of clinical information assess the existing evidence with variable rigour, and usually do not describe the quality of the evidence underpinning their statements. To answer our question we analysed original research by searching the MEDLINE ${ }^{\circledR}$ database.

Because of the erratic manner in which articles dealing with this topic are indexed in the MEDLINE ${ }^{\circledR}$ database, finding the relevant evidence was laborious and required expertise. We searched the literature by combining free-text and MeSH terms in PubMed (http://www.ncbi.nlm.nih.gov/entrez/query.fcgi), using "seizure recurrence", "unprovoked seizure*", "first seizure", "single seizure*", "prognosis" and "anticonvulsant*". This yielded several hundred articles of which 18 seemed relevant. ${ }^{1-18}$ Of particular interest were a meta-analysis of 16 articles dealing with prognosis in adults with single seizures, ${ }^{4}$ and two randomized controlled trials (RCTs) of anticonvulsants versus observation of patients with a first seizure. ${ }^{1,8}$ One of the RCTs is a large multicentre study. ${ }^{1}$ Because meta-analyses of relevant studies and large RCTs are the strongest type of evidence to answer our question about prognosis and treatment, we will focus on these two pieces of external evidence. ${ }^{1,4}$

\section{Critically appraising and applying the evidence}

\footnotetext{
* This is standardized truncation nomenclature in PubMed
}

The next step is to assess whether the evidence is valid (ie, whether it is close to the truth and we can trust it) and whether we can apply it to our patient. This task is made easier by following published "Users' guides" to appraise the medical literature, ${ }^{19-21}$ which can be found in full text at www.cche.net/principles/main.asp.

\section{Meta-analysis of prognosis after a first unprovoked seizure ${ }^{4}$}

We can appraise this study following published guides to assess the validity of review articles, ${ }^{21}$ as follows:

\section{Is this Meta-analysis valid?}

\section{1) Did the meta-analysis address a focused clinical question?}

A clear statement of the questions being addressed helps to determine whether the meta-analysis is relevant to the care of our individual patient. This meta-analysis addresses the specific question of risk of recurrence after a first unprovoked seizure. ${ }^{4}$

\section{2) Were the methods used to find and select articles appropriate?}

This is important because differences in seizure types, age, cause of seizures and type of follow-up may give different results in meta-analyses that appear to ask the same question. This metaanalysis has a broad, clearly stated inclusion criterion, i.e., articles addressing the risk of recurrence following a first unprovoked seizure. The search for articles to include must be thorough and avoid publication and researcher bias. Normally, this includes a bibliographic database like MEDLINE ${ }^{\circledR}$, the bibliographies of the relevant papers, and personal contact with experts in the area. The literature search in this meta-analysis is reasonably complete but not exhaustive; the authors used the MEDLINE $^{\circledR}$ database and references of relevant papers, but did not look at unpublished data or other databases.

\section{3) Was the validity of the studies included in the meta-analysis} appraised?

The authors assessed whether "seizures" were clearly defined and excluded one paper on this basis. They distinguished between retrospective and prospective case ascertainment and the problems with retrospective studies were clearly stated. Because results vary significantly depending on whether patients are enrolled at the time of a first seizure (prospectively) or until they presented with a recurrence (retrospectively), a separate analysis is provided for prospective and retrospective studies. Recognizing that duration of follow-up is important in studies about prognosis, the authors only included studies with a minimum follow-up of one to two years.

4) Was assessment of studies reproducible and were the results similar from study to study?

Judgement is required to decide which articles to include, how valid they are and which data to extract. Having more than one person assess each decision decreases the chance for error or bias. In this meta-analysis, we are not told whether more than one reviewer assessed studies independently. Therefore, we do not know whether this aspect of the analysis is reliable. When the results of individual studies included in a meta-analysis are very dissimilar, there is a good chance that differences in study design, patient population, or outcome measurement, account for this heterogeneity. The authors acknowledge that although meta- 
analyses including articles with more homogeneous results are desirable, this was not feasible in this review. They tabulate discrepancies in seizure recurrence (ranging from $23 \%$ to $71 \%$ ), and suggest possible explanations, eg., different inclusion criteria and prospective vs. retrospective studies.

Overall, our critical appraisal of this meta-analysis reveals that it is valid and we can go on to assess its results.

\section{What are the results of this meta-analysis and can I apply them to my patient?}

From 13 studies including 1,930 patients, the authors calculated a pooled, overall risk of recurrence of $46 \%(95 \% \mathrm{CI}=$ $44 \%, 49 \%$ ) at two to five years. The overall two-year risk was $42 \%(95 \% \mathrm{CI}=39 \%, 44 \%)$, and it was lower in five prospective $(40 \%)$ than in seven retrospective $(52 \%)$ studies. The risk of recurrence was almost identical in children and adults, ie., $43 \%$ $(95 \% \mathrm{CI}=38 \%, 47 \%)$ in both. About $80 \%$ of the five-year risk is realized by two years. The authors also provide a useful summary measure of comparative risk among various subgroups (the relative risk). This shows that seizure recurrence is almost twice higher among patients who have neurological abnormalities, or an EEG with epileptiform abnormalities. Pooled relative risks could not be estimated separately for generalized and partial seizures, but individual studies showed higher recurrence rates for partial than generalized seizures (relative risk 1.3-1.6). In summary, the results of a subgroup of articles in the meta-analysis are applicable to a young, previously healthy individual with a first unprovoked generalized seizure, whose two-year risk of recurrence is about $40 \%$.

\section{Randomized controlled trial of anticonvulsants for the first seizure $^{1}$}

In this multicentre study, 397 patients of all ages presenting within seven days of their first unprovoked generalized tonic clonic seizure, were randomized to immediate treatment with AEDs (phenytoin, phenobarbital, valproate or carbamazepine) or to treatment only after seizures recurred. We can appraise it following published guides to assess the validity of studies about therapy. ${ }^{19,20}$

\section{Are the results of the study valid?}

A reasonable first question to ask is whether the results of the study have been influenced by systematic error or bias leading to a false conclusion. To assess this, one needs to know the following:

1) Were patients randomly assigned to treatment, and was the randomization list concealed?

Randomization is a central aspect of therapeutic trials. Its relevance is illustrated by the discrepancy between all nonrandomized and the one RCT regarding the effects of AEDs on seizure recurrence. ${ }^{1}$ Only the RCT found a favourable impact of AEDs on risk of recurrence, suggesting a bias towards treating only high-risk patients in non-randomized reports. The study randomized 468 eligible patients to treatment or no treatment with AEDs after a first unprovoked seizure. With this number, the authors were $80 \%$ certain to find a $50 \%$ difference in seizure recurrence between the groups. Baseline clinical characteristics were similar in both groups.

Concealed randomization refers to taking adequate measures to hide the allocation to study groups from those responsible for enrolling patients. Therefore, it protects from selection bias. Lack of concealment is a potent source of bias. Trials in which randomization is not concealed may overestimate benefits by about $40 \% .{ }^{22}$ Although the authors of this trial do not explicitly describe concealment, this can be assumed by the multicentre nature of the study.

2) Were all patients accounted for at the end of the study, and were they analysed within the group to which they were randomized?

This is important because patients who complete a study differ systematically from those who do not complete it. The authors account for all patients screened (840), eligible (515), randomized (468), incorrectly randomized (32), and lost followup (14), as well as the reasons for exclusions. We are not told how similar eligible but not randomized patients were to those actually randomized, but the description of included patients helps us know how similar they are to our patient. Although it would be useful to know the features of the 46 patients ( $10 \%$ of sample) who were either randomized incorrectly or lost followup, the proportion of patients with missing data is small and does not invalidate the results.

The analysis was done when 397 patients had at least some follow-up. Patients were analysed within the groups to which they were randomized, regardless of compliance (intention to treat). This strategy preserves the unbiased comparison provided by randomization. A substantial number of patients (up to 20\%) randomized to treatment discontinued it at some point and the authors provide the seizures recurrence for the group who actually continued to take anticonvulsants.

3) Were clinicians, researchers and patients blinded to treatment and were both groups treated equally, aside from the experimental intervention?

Blinding is essential because expectations of patients and clinicians about the efficacy of interventions may distort the reporting and the measurement of outcomes. Neither patients nor clinicians were blinded in this trial. ${ }^{1}$ Conceivably, this could bias the results in favour of treatment for several reasons. First, seizures could be under-called in the treatment group (particularly if seizures are clinically subtle). Second, although the study aimed at similar follow-up in both patient groups, the potential for differential intensity in follow-up exists when clinicians are not blinded to the treatment group. Finally, we do not know exactly what types of seizures were judged as a recurrence, although it is implied that all seizure types were counted. The authors acknowledge this and partly alleviate our concerns by stating that the first recurrence in all patients included generalized tonic clonic seizures. In summary, although blinding would have strengthened the study, its absence does not invalidate it.

Overall, the study is valid. We can now examine its results and how they apply to our patient.

\section{What are the results and are they likely to help in this patient's care?}

The cumulative risk of recurrence for untreated and treated patients was $51 \%$ and $25 \%$ respectively at two years. This baseline risk (51\%) is in keeping with Berg's ${ }^{4}$ meta-analysis on prognosis after a first seizure. The absolute reduction in the risk 
of seizure recurrence with treatment is $26 \%$. This means that four patients with a first unprovoked generalized seizure need to be treated in order to avoid one recurrence in two years. ${ }^{23}$ This is calculated by dividing one over the difference of recurrence in untreated (0.5) and in treated (0.25) patients, ie., $1 / 0.25=4$. This is a clinically significant result and compares favourably with other interventions. For example, 10 patients need to undergo carotid endarterectomy in order to prevent one stroke. ${ }^{24}$ The hazard ratio (a comparative measure of recurrence between the two groups) and its $95 \%$ confidence intervals show that the risk of relapse in untreated subjects is two to four times higher than in treated patients. In per-protocol analyses (only including patients who actually took the anticonvulsants), the recurrence rate was even lower (15\%).

An analysis of the patients'characteristics in this trial does not reveal a compelling reason why the results could not be applied to our 25-year-old female patient with a first unprovoked seizure.

\section{TO TREAT OR NOT TO TREAT}

Overall, current evidence suggests that patients with a first, unprovoked seizure have a risk of recurrence of $50 \%$ on the average, and that treatment with anticonvulsants reduces the risk of recurrence to about $25 \%$ at two years, with a number needed to treat of four. This distilled evidence is informative and useful in our decision making process. However, it is not the role of EBC to make clinical decisions, but rather to inform skilled clinicians who can judiciously and conscientiously incorporate the evidence with the patients' particular circumstances and values. Because the final decision involves the informed patient, before recommending AEDs, the patient and the clinician should consider potential harm and side effects, costs, the labelling effect of starting AEDs, and the impact on quality of life. The patient's occupation, aversion to the risk of recurrent seizures, and requirement of a driver's license are other relevant considerations. Little conclusive evidence exists on long-term outcomes and impact of therapy on the subsequent development of epilepsy. However, we know that long-term mortality is not increased in those with a first unprovoked generalized seizure, ${ }^{14}$ and some evidence is accruing that treatment of the first as opposed to the second seizure does not prevent the development of subsequent epilepsy. ${ }^{13}$ In summary, management of patients with a first seizure requires good communication between a skilled clinician armed with the best external evidence and an informed patient and family.

A one-page EBC summary deriving from this analysis, ie., a CAT, is presented in the appendix. The CAT's title is the declarative answer to the question addressed. The components of a CAT are: 1) the declarative answer to the clinical question, 2) the clinical question, 3) the literature search strategy, 4) one to four clinical bottom lines in point-form, 5) a description of the evidence and of the actual data, 6) comments, and 7) references. If the CAT is peer-reviewed, this should be stated.

\section{REFERENCES}

1. First Seizure Trial Group. Randomized clinical trial on the efficacy of antiepileptic drugs in reducing the risk of relapse after a first unprovoked tonic-clonic seizure. Neurology 1993; 54: 478-483.
2. Annegers JF, Shirts SB, Hauser WA, Kurland LT. Risk of recurrence after an initial unprovoked seizure. Epilepsia 1986; 27: 43-50.

3. Baumhackl U, Billeth R, Graf M. Type-specific diagnostic analysis of first epileptic seizure in adults. Eur Neurol 1994; 34 Suppl 1: 71-73.

4. BergAT, Shinnar S. The risk of seizure recurrence following a first unprovoked seizure: a quantitative review. Neurology 1991; 41: 965-972.

5. Bora I, Seckin B, Zarifoglu M, et al. Risk of recurrence after first unprovoked tonic-clonic seizure in adults. J Neurol 1995; 242: 157-163.

6. Elwes RDC, Chesterman P, Reynolds EH. Prognosis after a first untreated tonic-clonic seizure. Lancet 1985; 752-753.

7. Forsgren L, Bucht G, Eriksson S, Bergmark L. Incidence and clinical characterization of unprovoked seizures in adults: a prospective population-based study. Epilepsia 1996; 37: 224-229.

8. Gilad R, Lampl Y, Gabbay U, Eshel Y, Sarova-Pinhas I. Early treatment of a single generalized tonic-clonic seizure to prevent recurrence. Arch Neurol 1996; 53: 1149-1152.

9. Gupta SK, Satishchandra P, Venkatesh A, Subbakrishna DK. Prognosis of single unprovoked seizure. J Assoc Physicians India 1993; 41: 709-710.

10. Hart YM, Sander JW, Johnson AL, Shorvon SD. National General Practice Study of Epilepsy: recurrence after a first seizure. Lancet 1990; 336: 1271-1274.

11. Hauser WA, Anderson VE, Loewenson RB, McRoberts SM. Seizure recurrence after a first unprovoked seizure. N Engl J Med 1982; 307: 522-528.

12. Hopkins A, Garman A, Clarke C. The first seizure in adult life. Value of clinical features, electroencephalography, and computerised tomographic scanning in prediction of seizure recurrence. Lancet 1988; 1: 721-726.

13. Musicco M, Beghi E, Solari A, Viani F. Treatment of first tonicclonic seizure does not improve the prognosis of epilepsy. First Seizure Trial Group (FIRSTGroup). Neurology 1997;49:991-998.

14. Olafsson E, Hauser WA, Gudmundsson G. Long-term survival of people with unprovoked seizures: a population- based study. Epilepsia 1998; 39: 89-92.

15. Tardy B, Lafond $\mathrm{P}$, Convers $\mathrm{P}$, et al. Adult first generalized seizure: etiology, biological tests, EEG, CT scan, in an ED. Am J Emerg Med 1995; 13: 1-5.

16. van Donselaar CA, Geerts AT, Schimsheimer RJ. Idiopathic first seizure in adult life: who should be treated? Br Med J 1991; 302: 620-623.

17. van Donselaar CA, Schimsheimer RJ, Geerts AT, Declerck AC. Value of the electroencephalogram in adult patients with untreated idiopathic first seizures. Arch Neurol 1992;49:231-237.

18. Wolf P. Non-medical treatment of first epileptic seizures in adolescence and adulthood. Seizure 1995; 4: 87-94.

19. Guyatt GH, Sackett DL, Cook DJ. Users' guides to the medical literature. II. How to use an article about therapy or prevention. A. Are the results of the study valid? Evidence-Based Medicine Working Group. JAMA1993; 270: 2598-2601.

20. Guyatt GH, Sackett DL, Cook DJ. Users' guides to the medical literature. II. How to use an article about therapy or prevention. B. What were the results and will they help me in caring for my patients? Evidence-Based Medicine Working Group. JAMA 1994; 271: 59-63.

21. Oxman AD, Cook DJ, Guyatt GH. Users' guides to the medical literature. VI. How to use an overview. Evidence-Based Medicine Working Group. JAMA1994; 272: 1367-1371.

22. Chalmers TC, Celano P, Sacks HS, Smith H. Bias in treatment assignment in controlled clinical trials. N Engl J Med 1983; 390: 1358-1361.

23. Laupacis A, Sackett DL, Roberts RS. An assessment of clinically useful measures of the consequences of treatment. N Engl J Med 1988; 318: 1728-1733.

24. North American Symptomatic Carotid Endarterectomy Trial Collaborators. Beneficial Effect of Carotid Endarterectomy in Symptomatic Patients with High-grade Carotid Stenosis. N Engl J Med 1991; 325: 445-453. 


\section{APPENDIX (CAT)}

\section{Overall, the risk of seizure recurrence after a first unprovoked generalized motor seizure was $40 \%$ to $50 \%$ at two years. Treatment with anticonvulsants decreased this risk to $25 \%$.}

\section{Clinical question}

In a healthy adult with a first, unprovoked generalized tonic clonic seizure and normal neurological examination, what is the risk of seizure recurrence, and do anticonvulsants decrease the risk of recurrence?

\section{Literature search}

Combination of free-text and $\mathrm{MeSH}$ terms in PubMed: "seizure recurrence", "unprovoked seizure*", "first seizure", "single seizure*", "prognosis" and "anticonvulsant*".

\section{Clinical Bottom Lines}

- Overall, the risk of seizure recurrence after a first, unprovoked generalized motor seizure is $42 \%(95 \% \mathrm{CI}=39 \%, 44 \%)$ at two years, and $46 \%(95 \% \mathrm{CI}=44 \%, 49 \%)$ up to five years.

- Neurological abnormalities or epileptiform activity in the EEG double the risk of recurrence.

- Anticonvulsants (Phenytoin, Carbamazepine, Valproate, or Phenobarbital) decreased the risk of recurrence to $25 \%$, with NNT $=4(95 \% \mathrm{CI}=3,6)$ at two years.

\section{The Evidence}

1. Meta-analysis of 1,930 patients in 13 studies of prognosis after a first unprovoked generalized motor seizure, with subgroup analyses by type of study (retrospective, prospective), age (adults, children), and assessment of risk factors.

2. Randomized trial of 468 patients with a first unprovoked generalized seizure assigned to immediate anticonvulsant treatment or to treatment only if seizures recurred. Analysis was by intention to treat at two years.

\author{
Data Description \\ Meta-analysis ${ }^{1}$
}

\begin{tabular}{lcc}
\hline & Time (years) & Pooled risk \% (95\% CI) \\
Overall & 1 to 5 & $51(49,53)$ \\
Overall & 2 & $42(39,44)$ \\
Prospective studies & 1 to 5 & $40(37,43)$ \\
Retrospective studies & 1 to 5 & $52(49,55)$
\end{tabular}

\section{Randomized Trial}

\begin{tabular}{lccc}
\hline Time & $\begin{array}{c}\text { Seizure free, } \\
\text { Treated }(\%)\end{array}$ & $\begin{array}{c}\text { Seizure free, } \\
\text { Untreated }(\%)\end{array}$ & $\begin{array}{c}\text { NNT } \\
(\mathbf{9 5} \% \mathbf{C I}) *\end{array}$ \\
3 months & 93.4 & 82.2 & $10(8,15)$ \\
6 months & 92 & 71.8 & $7(5,10)$ \\
12 months & 83 & 59.4 & $5(4,7)$ \\
24 months & 74.7 & 49.2 & $4(3,6)$
\end{tabular}

* NNT $=$ number needed to treat; this was obtained from data in the article.

\section{Comments}

Both studies are valid and have minor methodological problems. In the meta-analysis, the authors do not describe the reliability of article selection and data abstraction. Although there is no statistical measure of heterogeneity to assess whether the results of the studies can be combined, the authors examine relevant sources of variability. The randomized trial is not blinded and quality of life is not considered. The risk of recurrence after a first seizure is substantial (around 50\%) and it is significantly decreased by anticonvulsants. However, the decision to treat should consider factors such as side effects, costs, the labelling effect of starting AEDs, impact on quality of life, the patient's occupation, aversion to the risk of recurrent seizures, and requirement of a driver's license.

\section{REFERENCES}

1. BergAT, Shinnar S. The risk of seizure recurrence following a first unprovoked seizure: a quantitative review. Neurology 1991; 41: 965-972.

2. First Seizure Trial Group. Randomized clinical trial on the efficacy of antiepileptic drugs in reducing the risk of relapse after a first unprovoked tonic-clonic seizure. Neurology 1993; 54: 478-483.

CAT created by: Dr. Samuel Wiebe. Date: September 2001. This CAT was peer-reviewed. 